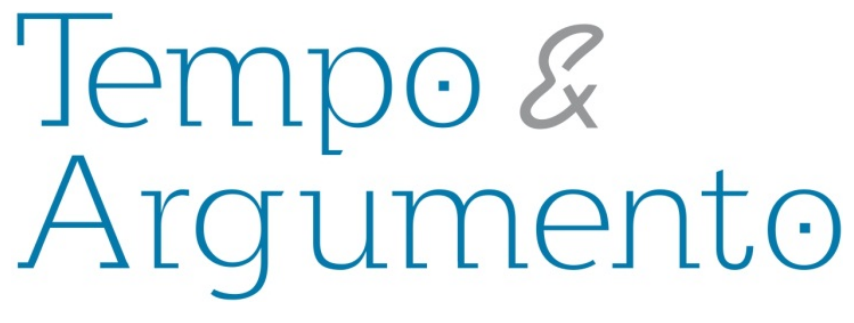

\title{
Le refus du secret : exercice de droits et accès à des documents publics. ${ }^{1}$
}

\begin{abstract}
Résumé
A partir de situations relativement récentes et polémiques produites par la mise à disposition, sur Internet (à travers WikiLeaks), d'une grande quantité de documents secrets appartenant à des instances gouvernementales de divers pays, on discute des aspects historiques concernant l'accès aux documents d'archives à caractère public, leur utilisation pour la production de connaissance historique et leurs intérêts pour l'exercice de la citoyenneté et la consolidation de pratiques démocratiques. Il s'agit aussi, brièvement, du débat archivistique autour de l'évaluation de documents d'archives et leur rapport avec le thème de l'accès aux archives.
\end{abstract}

Mots-clés: Archives publiques. Accès. Évaluation. Citoyenneté. Histoire.

\author{
Janice Gonçalves \\ Docteur en Histoire Sociale. \\ Professeur d'Histoire, \\ Universidade do Estado \\ de Santa Catarina. \\ janice_goncalves@hotmail.com
}

Traduction

Fernando Coelho

Doctorant en Linguistique,

Universidade Federal

de Santa Catarina

zeffiretto@gmail.com

\section{Pour citer cet article :}

GONÇALVES, Janice. Le refus du secret : exercice de droits et accès à des documents publics.

Revista Tempo e Argumento. Florianópolis, v. 5, n.9, jan./jun. 2013. p. 244 - 265.

\footnotetext{
${ }^{1}$ Version augmentée et mise à jour du texte présenté à la table ronde "A prática da confidencialidade e o direito de acesso a documentos produzidos pelo poder público", qui a fait partie de la programmation du $\mathrm{XVI}^{\text {ème }}$ Congresso Brasileiro de Arquivologia - "O lugar do arquivo". Ce congrès a eu lieu à Santos, São Paulo, entre le 24 et le 27 août 2010.
} 


\section{The refusal of secrets: exercise of rights and access to public archives}

\section{A recusa do segredo: exercício de direitos e acesso a documentos públicos}

\begin{abstract}
Thousands of secret government registers relative to many countries were published in recent years at the Internet through WikiLeaks. This polemic action gives the opportunity to discuss, in this article, some historical aspects of the access to public archival documents, their use for the production of historical knowledge and their relevance to citizenship and to consolidate democratic practices. The debate about archival appraisal and its relationship to archival documents access are also briefly discussed.
\end{abstract}

Keywords: Public archives. Access. Appraisal. Citizenship. History.

\begin{abstract}
Resumo
A partir de situações relativamente recentes e polêmicas de disponibilização, na Internet (por meio do WikiLeaks), de grande volume de documentos sigilosos produzidos no âmbito de instâncias governamentais e referentes a diversos países, são discutidos aspectos históricos que envolvem $\mathrm{O}$ acesso à documentação arquivística de caráter público, sua utilização para a produção de conhecimento histórico e sua relevância para o exercício da cidadania e a consolidação de práticas democráticas. Aborda-se ainda, brevemente, o debate arquivístico acerca da avaliação de documentos de arquivo e sua relação com o tema do acesso aos arquivos.
\end{abstract}

Palavras-chave: Arquivos públicos. Acesso. Avaliação. Cidadania. História.

À la fin du mois de juillet 2010, les médias ont signalé la mise à disposition, sur un site Internet, de plus de mille documents confidentiels relatifs aux actions militaires nordaméricaines des six années précédentes. La lecture du «Afghan War Diary » - titre donné 
à l'ensemble de ces documents par WikiLeaks, site responsable de leur divulgation révélait une partie significative du quotidien de la guerre, absente de la presse. ${ }^{2}$ Polémique, l'initiative a suscité des critiques du gouvernement des EUA et de diverses institutions, surtout du fait que les documents identifiaient des collaborateurs des forces de l'occupation en Afghanistan, ce qui pourrait mettre leurs vies en risque. D'autre part, les manifestations favorables ont été également expressives, en saluant l'importance des moyens de communications pour la défense des droits à l'information et à la liberté d'expression.

Au «Afghan War Diary » succéderaient de nouvelles publications frappantes et polémiques promues par WikiLeaks: en octobre 2010, environ 400 mille documents secrets portant sur l'occupation en Irak par les EUA et, en novembre, plus de deux cent mille documents diplomatiques produits par des ambassades nord-américaines. La divulgation ébranlerait les gouvernements concernés (surtout celui des États-Unis), mais WikiLeaks n'en sortirait pas indemne : il aurait des problèmes à maintenir financièrement ses actions (des institutions financières ont bloqué des virements bancaires et des dons moyennant carte de crédit faits à WikiLeaks), supporteurs connus subiraient des représailles et son éditeur, Julian Assange, serait objet d'un procès et emprisonné sous accusation de crimes sexuels prétendument commis en Suède.

À la suite des développements du procès pour abus sexuel et viol, Assange a été maintenu en prison domiciliaire à Londres, entre décembre 2010 et mai 2012, jusqu'à la confirmation de son extradition vers la Suède. L'extradition cependant n'a pas été effectuée parce qu'Assange a demandé l'asile à l'ambassade de l'Equateur à Londres (ASSANGE, 2013, p. 37-41 e 161-163).

Le site Internet WikiLeaks, accessible depuis 2006, est la face visible et le principal instrument d'action d'une organisation dont l'objectif fondamental est de rendre disponibles des informations qui, en dépit de leur intérêt public, ont été systématiquement occultées. Pour les obtenir et les disséminer, WikiLeaks compte sur un

\footnotetext{
2 Le "Afghan War Diary" a été mis à disposition, d'abord, sur le site <http://wardiary.wikileaks.org/> et en 2013 il peut être lu sur <http://wikileaks.org/wiki/Afghan_War_Diary,_2004-2010>.
} 

fait en envisageant trois principes : «la défense de la liberté d'expression et de presse, le perfectionnement de nos registres historiques en commun et le support aux droits de tous les peuples de créer histoire nouvelle. » En plus, on met en valeur la divulgation d'un grand nombre de documents qui, de façon incisive, prouvent les dénonciations: par conséquent, selon les responsables de WikiLeaks, « lecteurs et aussi historiens peuvent voir l'évidence de la vérité ».3

Il est convenable de poser la question à propos des signifiés de cette « histoire nouvelle » dont le droit de produire est affirmé par WikiLeaks. Nouvelle, peut-être, parce que récente? Le biais politique de ses actions autorise à penser à une autre perspective de «nouveauté »: des récits historiques différents de ceux qui sont hégémoniques, en général construits à partir de ce qui est passé par les ciseaux de la censure, qu'on a permis de révéler et qui est devenu ouvert à la consultation publique. Une « histoire nouvelle » traverserait ainsi les mailles de l'occultation. L'impressionnant volume de documents confidentiels rendus disponibles sur le site Internet WikiLeaks, depuis sa création, met en échec, donc, la documentation effectivement disponible à la consultation dans les institutions archivistiques publiques et la possibilité de construire, à partir d'elle, une histoire qui dépasse effectivement les limites imposées par des intérêts de groupes qui contrôlent soit des sphères étatiques, soit l'accès aux registres documentaires générés par elles. Plus que cela : à une époque qui valorise la vitesse et désire tout scruter (de préférence en «temps réel »), est inévitable que la divulgation frappante, à tous les usagers d'Internet, de milliers de documents, une partie significative d'eux étant relative à des actions militaires (qui, en plus d'un sens, ont été très couteux), renvoie à la morosité et opacité des institutions gouvernementales, y comprises les institutions archivistiques publiques.

Les doutes surgissent avec force : enfin, à quels documents publics, en fait, les citoyens ont-ils accès? Dans des circonstances dans lesquelles l'accès à des documents

\footnotetext{
${ }^{3}$ Cf.: <http://wikileaks.org/About.html >. Consulté le 13 mai 2013.
} 
d'archives publics puisse changer les directions d'une guerre, altérer un réseau d'alliances politiques, réduire le support à un gouvernement ou nuire un projet de domination, quelle est la probabilité que la consultation à ces documents soit permise et réalisée de façon agile? La législation régulatrice de l'accès, ainsi que les instances responsables de son observance, suffiraient-elles à garantir que la documentation en question soit mise à disposition dans une institution archivistique publique à tout citoyen intéressé ? Comment éviter que la législation qui bloque l'accès aux documents au nom de la "sécurité nationale » et qui, en même temps, cherche le traitement étique des informations concernant les individus, serve pragmatiquement à des intérêts politiques moins nobles, plus restreints et immédiats? Observant fidèlement cette législation, ces institutions archivistiques ne seraient-elles pas complices de la logique du secret, plutôt qu'assises de la transparence des actions gouvernementales et instruments de la démocratie? Ou encore, comme a questionné Cassie Findlay : «maintenant, puisque la technologie nous permet d'éliminer beaucoup des obstacles de l'héritage de la gestion des documents basée sur papier, est-il possible aux archives de revendiquer leur position au cœur d'une démocratie saine ? » (FINDLAY, 2013, p. 7-8).

\section{Ce qu'on occulte, ce qu'on montre?}

Comment penser le rapport entre gouvernants et gouvernés, par le moyen des archives ?

Les documents gouvernementaux sont produits en fonction de l'intégration des gouvernements avec les gouvernés : soit parce que les gouvernants, ou leurs préposés, se tournent vers les gouvernés pour les identifier, comprendre leurs profils et leurs demandes et, à la limite, les contrôler, soit parce que les gouvernés répondent à des interventions et en suscitent d'autres. Par conséquent, à côté, par exemple, de listes qui essayent de faire un recensement de la population ou enregistrer les propriétaires des immeubles d'un territoire donné, on peut trouver, aux archives gouvernementales, toutes sortes de demandes, faites par les citoyens aux autorités. Dans la législation brésilienne, les demandes des citoyens ont été clairement prévues dans la plupart des 
documents constitutionnels, sous forme de la garantie de deux droits : celui de pétition aux pouvoirs publics (à la défense d'autres droits ou contre des illégalités et des abus) ${ }^{4}$ et celui de contestation (visant l'annulation d'actes qui puissent léser le patrimoine public) ${ }^{5}$. Une fois exercés, les droits de pétition et de contestation ont produit (et peuvent produire encore) des documents qui, envoyés aux instances publiques compétentes, ont été (et, à présent, doivent être) intégrés aux ensembles de documents respectifs.

En plus, l'étroite proximité entre les documents provenant des organes publics et la population vers laquelle ils sont dirigés a fait que l'accès aux informations des documents d'archives publics soit prévu par la loi dans des moments historiques très antérieurs à celui des débats modernes autour de la citoyenneté et de l'intérêt public. L'archiviste italien Elio Lodolini (1993, p. 247) souligne, par exemple, que la sollicitation, par des particuliers, de copies de documents avec foi publique, pour des fins juridiques, « est parvenue à être un fait normal », durant l'Empire Romain; d'autres chercheurs ajoutent que ces copies étaient obtenues moyennant une demande à l'empereur, qui les autorisait par écrit (SILVA et al, 1999, p. 64). Lodolini $(1993,247)$ met en valeur aussi l'accès aux archives des communes médiévales dans la Péninsule Italique.

Dans la législation brésilienne, avant que le droit d'accès à l'information fût pleinement reconnu (comme il l'a été, par la Constitution Fédérale de 1988, à l'article 5, incise XIV), le droit à l'obtention de certificats d'institutions publiques, à la défense de la garantie d'autres droits ou à l'obtention d'éclaircissements, a été soutenu dans les textes

\footnotetext{
${ }^{4}$ Le droit de pétition était prévu dans les constitutions de 1824 (Art. 179, incise XXX), 1891 (Art. 72, paragraphe 9), 1934 (Art. 113, paragraphe 10), 1937 (Art. 122, paragraphe 7), 1946 (Art. 141, paragraphe 37), 1967 (Art. 150, paragraphe 31, dans la rédaction originale, et Art.152, paragraphe 31, avec la rédaction modifiée de 1969), 1988 (Art. 5, incise XXXIV, alinéa a). La Constitution de 1824 déterminait : "Todo Cidadão poderá apresentar por escrito ao Poder Legislativo e ao Executivo reclamações, queixas, ou petições, e até expor qualquer infração da Constituição, requerendo perante a competente autoridade a efetiva responsabilidade dos infratores." [Traduction : Tout Citoyen pourra présenter par écrit au Pouvoir Législatif et au Pouvoir Executif des réclamations, des plaintes ou des pétitions, et il pourra aussi exposer quelque infraction de la Constitution, requérant devant l'autorité compétente l'effective responsabilité des infracteurs.] La Constitution de 1937 soulignait plus synthétiquement que le droit " de représentation ou pétition devant les autorités" était basé sur la défense "de droits ou de l'intérêt général" . (CAMPANHOLE, 1994).

5 Prévu dans les constitutions de 1934 (Art. 113, paragraphe 38), 1946 (Art. 141, paragraphe 38), 1967 (Art. 150, paragraphe 31, dans la rédaction originale, et Art.152, paragraphe 31, avec la rédaction modifiée de 1969), 1988 (Art. 5, incise LXXIII). Selon la Constitution de 1988, peuvent être également contestés les actes nuisibles à la moralité publique, à l'environnement et au patrimoine historique et culturel. (CAMPANHOLE, 1994).
} 
constitutionnels de 1934 (Art. 113, paragraphe 35), 1946 (Art. 141, paragraphe 36), 1967 (Art. 150, paragraphe 34, et art. 153, paragraphe 35 dans la rédaction modifiée de 1969). ${ }^{6}$ Le type d'éclaircissements sollicités n’a pas été prévu avec précision par les textes constitutionnels - dans celui de 1967, on ne parle que d'«éclaircissements de situations », sans d'autres détails; dans celui de 1988, tels éclaircissements apparaissent en référence à des situations d'intérêt personnel. Dans certains cas, particulièrement dans les textes constitutionnels de 1934 et 1964 , des délimitations ont été faites en ce qui concerne la sauvegarde de l'intérêt public : dans la Constitution de 1934, l'éclaircissement sollicité devrait se référer aux "affaires publiques », à l'exception des cas où l'intérêt public imposerait « secret ou réserve »; dans celle de 1946, l'éclaircissement devrait être relatif à des « affaires administratives », à moins que l'intérêt public n'imposât la confidentialité.

Même si, de façon restreinte, et au moins à la lettre de la loi, le droit d'obtenir des certificats, dans une certaine mesure, a permis (et permet encore) l'accès aux informations relatives aux affaires publiques. II s'agit, cependant, d'un accès indirect, qui suppose l'intermédiation de l'agent gouvernemental qui élaborera le certificat. La logique des relations de l'Etat avec la société civile permet d'observer quelques motivations pour cela. D'un côté, le besoin de sauvegarder les droits de particuliers garantis par documents gouvernementaux est reconnu; droits ponctuels sont alors reconnus, spécifiquement ceux concernant les individus qui sollicitent des certificats. Dans ces cas, l'accès (indirect) aux documents d'archives n'implique pas la reconnaissance d'un droit collectif à l'information. Considérant le profil probable de ceux qui demanderaient le plus habituellement ces informations d'intérêt individuel, auteurs tels qu'Alberch Fugueras et Cruz Mundet ont souligné le caractère élitiste de l'accès aux archives dans la plupart de leur histoire :

\footnotetext{
${ }^{6}$ Il a été absent de la Constitution de 1937. II faut ajouter que la Constitution de 1988 a prévu le droit de recevoir, des organes publics, non seulement des information concernant les citoyens, individuellement (informations «de leur intérêt particulier »), mais aussi des information «d'intérêt collectif ou général » (Art. 5, incise XXXIII). De son côté, l'« habeas data » a renforcé et amplifié, simultanément, le droit de prendre connaissance des informations enregistrées dans des institutions gouvernementales qui soient relatives à chaque citoyen (car il permet la rectification de ces données. - Art. 5, incise LXXII). (CAMPANHOLE, 1994, p.10-13).
} 
Se representássemos em um segmento os aproximadamente 5.500 anos de existência dos documentos escritos, veríamos que, salvo os últimos trinta e cinco anos [em relação a 1999], no melhor dos casos, um pedacinho, todo o segmento estaria referido a uma constante histórica: o acesso aos documentos está reservado às elites no poder. Só com o desaparecimento das instituições do Antigo Regime a partir da Revolução Francesa (1789), seus papéis - já históricos e carentes de interesse para o Estado - começaram a ser postos à disposição dos pesquisadores; contudo, a informação estratégica, a que confere privilégios a quem a possui, continuava a estar ao serviço da gestão do poder e vedada aos cidadãos. (ALBERCH/CRUZ, 1999, p.100). ${ }^{7}$

Toutefois, en plus de promouvoir l'accès ponctuel et restreint aux documents, les certificats préservent, à principe, l'intégrité physique des documents et, par conséquent, leur valeur de preuve, en les protégeant d'altérations ou de dommages. Il convient de rappeler: les livres de registres créés en raison de la Loi de Terres de 1850, justement parce qu'ils ont maintenu leur valeur juridique, généralement ne permettent pas aux usagers la consultation directe, afin d'éviter des adultérations. Aux Archives Publiques de l'Etat de São Paulo, jusqu'à la décennie 1980, l'accès aux registres de terre était fait par le moyen de certificats. En raison des difficultés qu'il y avait pour la recherche historique, on a conçu, dans les années 1980, le projet d'inventaire analytique de ces registres, de façon à rendre disponibles, de manière ample et systématique, les informations d'intérêt pour les chercheurs, sans éliminer l'empêchement de la consultation directe (TESSITORE, 1986, p. 188-189). Il est clair que la solution trouvée pour amplifier l'accès, par le moyen d'un outil de description (inventaire analytique), a été proposée dans un moment où les possibilités de reproduction technique de la documentation étaient sensiblement plus distantes du quotidien des institutions archivistiques brésiliennes. Ce qu'on a pour objectif de retenir, cependant, à partir de l'exemple donné, c'est la préoccupation qu'a usuellement l'institution qui a la garde des documents d'archives par rapport à l'accès

\footnotetext{
${ }^{7}$ Traduction : Si nous représentions dans un segment les approximativement 5500 années d'existence des documents écrits, nous verrions que, sauf les dernières trente et cinq années (par rapport à 1990), dans le meilleur des cas, un petit morceau, tout le segment se référerait à une constante historique : l'accès aux documents est réservé aux élites au pouvoir. Ce n'est qu'à la disparition des institutions de l'Ancien Régime à partir de la Révolution Française (1789), leurs rôles - déjà historiques et sans intérêt pour l'Etat ont commencé à être mis à disposition des chercheurs; cependant, l'information stratégique, celle qui confère des privilèges a qui les possède, continuait à être à service de la gestion du pouvoir et interdite aux citoyens.
} 

documents, rapproche les citoyens des informations, quoique de façon limitée, en même temps que les éloigne de la documentation. Ce jeu de rapprochement et d'éloignement est présent de différentes manières, dans de différents moments historiques et sociétés, pénétrant les relations entre gouvernants et gouvernés, toujours sous tension, de part en part, par prérogatives et obligations, droits et devoirs. En raison de cela, les documents d'archives ont pu servir (et le peuvent encore) aux gouvernants, pour soutenir le soupçon, la surveillance, la répression et l'oppression. Par conséquent, les archives ont pu (et peuvent) être convoités (par qui exerce la domination ou la vise), ainsi que haïs (par qui souffre les effets de la domination).

Historiquement, gouvernants et gouvernés non seulement ont combattu pour contrôler et préserver les documents d'archives, mais aussi ils se sont efforcés de les éliminer. Guerres et révolutions sont des moments particulièrement significatifs pour des appropriations planifiées et des destructions intéressées. Les archivistes espagnols Alberch Fugueras et Cruz Mundet ont souligné la concentration, à Salamanque, d'archives enlevées de régions républicaines occupées par les troupes commandées par Franco, pendant la Guerre Civile Espagnole, les documents ayant été postérieurement utilisés pour les actions répressives du régime franquiste. Les mêmes auteurs montre la destruction de dix kilomètres de documents des Archives Centrales de Documents Anciens de Varsovie par l'armée de l'Allemagne pendant la Seconde Guerre, ainsi que la destruction de fonds, bibliothèques et musées promue par l'armée serbe dans des villes croates et bosniaques (ALBERCH/CRUZ, 1999, p. 97-98). Bruno Delmas (2006, p. 20) a enregistré la préoccupation spéciale, à la guerre entre Serbes et Croates dans les années 1990, de détruire des archives notariales, de façon à empêcher ou rendre difficile la preuve de l'identité civile et la revendication de droits, ce qui s'est aussi avéré à la Guerre du Liban de 1975. Similairement, dans la France révolutionnaire du XVIII ${ }^{\text {ème }}$ siècle, des documents qui attestaient des droits de nobles et d'ordres ecclésiastiques ont été systématiquement éliminés. 
Dans les sociétés occidentales, à partir du moment où la relation entre gouvernés et gouvernants a commencé à admettre les notions de bien commun, citoyenneté et intérêt public - donc et surtout à partir de la fin du XVIII ème siècle -, il y a eu une valorisation discursive graduelle des archives et une proposition de leur accès comme un droit collectif. Cependant, la garantie d'accès aux archives ne sera significativement affirmée, dans plusieurs pays, qu'après 1945, une fois que la reconnaissance du droit à l'information comme un droit fondamental a été consacré par la Déclaration Universelle des Droits de l'Homme, de $1948 .^{8}$ Le « droit à l'information gouvernementale » a été assuré dans plusieurs pays à partir de ce moment: en 1951, en Finlande; en 1966, aux Etats-Unis ; en 1970, en Danemark et Norvège ; en 1978, en France, Hollande et Espagne ; en 1982, en Australie, Nouvelle-Zélande et Canada; en 1985, en Colombie; en 1993, en Hongrie (DUCHEIN, 1983, p. 11 ; JARDIM, 1999, p.70-71).

Le droit à l'information, qui doit être exercé par l'ensemble des citoyens, exigerait que les gouvernements rendent compte à la société, sans occultation de procédures ou résultats d'actions. Mais, dans un contexte de l'implantation d'un nouvel ordre mondial, caractérisé par la bipolarisation entre les EUA et l'URSS et par ce qu'on appelle la « Guerre froide ", il est possible d'observer la difficile coexistence du droit à l'information et des services d'espionnage ou contre-espionnage, et, par conséquent, la prévalence d'une logique de secret au nom de la sécurité des Etats nationaux.

\section{Documents pour l'Histoire}

Il est certain que l'affirmation ou la défense d'un droit ne signifie pas son exercice sans entraves, qui est sujet à d'innombrables injonctions politiques et disputes entre groupes sociaux. Pour autant, l'importance de penser, ensemble avec le système juridique, les agents sociaux intéressés soit à l'ouverture des archives publiques aux citoyens soit au maintien de son interdiction. Il convient de faire ici une mention aux historiens.

\footnotetext{
${ }^{8}$ Il convient de souligner que, même avant la France, la Suède, à l'intérieur de préoccupations avec la liberté de presse, a garanti, en 1766, le libre accès aux documents oficiels à tous les citoyens suédois.
} 
L'accès ponctuel et restreint à des documents publics, comme celui qui est fait moyennant certificats ou copies (surtout de ces documents servant de preuve de droits individuels), ne pourrait pas satisfaire convenablement aux demandes de la recherche historique. Pas, au moins, à partir du XIX ${ }^{\text {ème }}$ siècle, moment où l'Histoire s'est constituée en tant que domaine disciplinaire et professionnel.

Les historiens du XIX ${ }^{\text {ème }}$ siècle n'ont pas été, certainement, les premiers à établir le lien entre recherche historique et archives. L'utilisation des documents d'archives comme un support de l'investigation à caractère historique (non seulement les gouvernementaux, mais aussi les privés, notamment les ecclésiastiques et familiales) a été possible à ceux qui en étaient proches, en raison de liens professionnels ou d'occupation ou de rapports personnels. Entre les anciens [peuples], cependant notamment entre les Grecs -, la valorisation de l'histoire contemporaine, vécue et témoignée par l'écrivain, n’a pas trouvé de répondant dans la valorisation de la consultation des archives ou de la claire identification, par les historiens, des documents éventuellement analysés. Comme a observé Paul Veyne (1987, p. 19), les auteurs anciens étaient, proprement, autorités sur le passé, et leurs récits formaient la « tradition » : ce qui avait été écrit était assumé comme vérité, laquelle pourrait éventuellement être complétée ou corrigée, ce qui faisait de la «vérité historique » une «vulgate consacrée par l'accord des esprits le long des siècles ». Si l'investigation était faite au présent et avec des témoignages cueillis directement (c'est-à-dire à partir de ce que l'investigateur avait vu ou écouté), les citations des sources n'étaient pas nécessaires - toujours selon Veyve (1987, p. 20-21), « un historien ancien [...] est lui-même source et document ».

Avant le XIX ${ }^{\text {ème }}$ siècle, aussi, la préoccupation avec la véracité et l'authenticité des sources peut être également détectée. En Europe, des investissements dans ce sens sont connus au moins depuis le XII ${ }^{\text {ème }}$ siècle ; toutefois, dans la période médiévale, même les documents diplomatiques subissent des altérations (amendements, suppressions) afin d'en faciliter la compréhension ou de les comptabiliser avec ce qui est affirmé "par quelque autorité ou par la tradition » (CAIRE-JABINET, 2003, p. 29-30). Il y a, cependant, grosso modo, une convergence d'interprétations dans le sens de situer entre les modernes la transition vers un nouveau modèle d'écriture historique, lié à l'intérêt 
accentué à établir des procédures pour détecter des documents authentiques et vérifier leur contenu de vérité. Plus amplement, François Hartog entend qu'il y a un « régime moderne d'historicité », installé «entre la fin du XVIII ${ }^{\text {ème }}$ siècle et le début du XIX ème , qui représente une rupture par rapport à l'« histoire maîtresse de la vie » des anciens: dans le régime moderne, l'histoire, entendue comme processus, cherche, plus que des exemples, des singularités, l'avenir étant sa dimension la plus déterminante, parce qu'elle donne du sens au passé - HARTOG, 1997, p. 8-11). Pour la construction de cette histoire de biais moderne, il y a eu la contribution d'érudits (dont les travaux fourniraient les bases de la «critique des documents"), ainsi que l'influence des processus argumentatifs développés dans les controverses théologiques et dans le domaine juridique, en plus des procédés provenant des pratiques des antiquaires, comme soulignent plusieurs auteurs (BLOCH, 2002, p. 91; CAIRE-JABINET, 2003, p.59, 64, 74-76; GINZBURG, 2002a, p. 71-76; GINZBURG, 2002b, p. 155-156; LE GOFF, 1996, p. 537; VEYNE, 1987, p. 23-24).

Au XIX ${ }^{\text {ème }}$ siècle, l'incorporation de la méthode de la critique des documents à l'intérêt de construction d'une histoire scientifique, en s'opposant aux philosophies de l'histoire, s'est articulée à la défense de la recherche empirique, du recensement systématique de documents et de leur analyse attentive (CAIRE-JABINET, 2003, p. 93-109; CARBONELL, 1986, p.104-125; REIS, 1999, p.11-16). En ce qui concerne les documents, ce projet a particulièrement valorisé la documentation archivistique publique, non seulement parce qu'elle servait plus favorablement aux intentions de construction des histoires nationales ou « histoires de la patrie » (se confondant, donc, avec l'histoire des Etats nationaux), mais aussi en raison des caractéristiques propres à cette documentation, qui présentait avec clarté les signes de son authenticité et, donc, donnait plus de fiabilité à son emploi et plus de solidité aux projets d'histoire scientifique qui étaient alors en vigueur (CAMARGO, 2009, p.433). Ainsi, les historiens ont obtenu, à partir du XIX ${ }^{\text {ème }}$ siècle, un rôle de plus en plus important dans l'élargissement de l'accès aux documents archivistiques publics: salles de consultation seraient installées dans les institutions européennes à la moitié du XIXème siècle (SILVA et al, 1999, p. 102). 
Elio Lodolini (1993, p. 250-251) cite, à ce propos, les cas des Archives nationales de France et des Archives de l'Allemagne, mais il souligne que la libération de la consultation pour la recherche historique était alors une exception plutôt qu'une norme. Des recherches sur les commencements de l'activité savante en Histoire, en Europe, indiquent que le contexte allemand, entre 1856 et 1915, rapprochait doctorants et leurs professeurs des «sociétés d'explorateurs, en s'aventurant dans des archives répandues partout, découvrant leur contenu et retournant pour dire à leur paires ce qu'ils avaient découvert. » (LAMBERT et al, 2011, p. 47). L'agitation autour de l'histoire a été aussi intense en Grande-Bretagne, où les salles de consultation sont bientôt apparues (la première date de 1858) et où les sociétés d'études historiques ont proliféré. (LAMBERT et al, 2011, p.48).

Le travail des historiens a été fondamental pour lier les archives à caractère permanent et les institutions archivistiques publiques responsables de leur garde (dotées de salles de consultations, horaires d'ouverture et instruments de recherche) à la recherche historique, qui, pendant le $X I X^{\text {ème }}$ siècle et jusqu'à la moitié du XXème siècle, était entendue fondamentalement comme une recherche dirigée, dans chaque pays, vers l'histoire de la nation (l'histoire «universelle » étant l'histoire de plusieurs nations). Disséminée, cette vision fixerait, dans le sens commun, l'association entre les archives publiques et leur « valeur historique », tendant à effacer d'autres valeurs qui puissent s'y ajouter, notamment leur fonction de base pour que les gouvernements rendent compte aux citoyens.

Au $X X^{\text {ème }}$ siècle, surtout dans son dernier quart, on a fait de fortes critiques à une historiographie forgée dans la construction d'histoires de la nation; critiques à leurs prétentions de scientificité «objective » et, inévitablement, critiques au privilège donné aux documents archivistiques publics comme base de la connaissance historique. Ce moment de critique et de crise a indiqué, entre d'autres besoins, celui de diversifier les sources sur lesquelles la production à caractère historique devrait s'appuyer. Dans certaines visions réductionnistes, les investigations historiques basées sur documentation gouvernementale ont été associées à des « histoires officielles »; s'opposer à une histoire officielle obligerait, ainsi, à un éloignement des documents publics. Parallèlement, on 

aux récits historiques, ainsi que la valorisation des documents et témoignages qui y sont associés. Si les archives publiques avaient été mises sous soupçon par les chercheurs professionnels, elles sont prises par des chercheurs amateurs anxieux de chercher des réponses à propos de leurs trajectoire familiale (ROUSSO, 1996, p. 85-91; HARTOG, 2013, p. 151-153).

La «vague mémorielle», avec ses correspondantes préoccupations avec le «devoir de mémoire» (presque toujours associées aux acquittements pendants par rapport à des événements traumatiques du $X X^{\text {ème }}$ siècle), a aussi atteint le domaine archivistique, et a suscité des réflexions sur ses fondements théoriques et méthodologiques, sur le rôle des professionnels d'archives et des institutions archivistiques (voir, par exemple : COOK, 1998 ; SCHWARTZ/COOK, 2004). La réflexion sur les signifiés et les procédures qui devraient être présents à l'évaluation archivistique est l'un des aspects les plus intéressants de ce débat.

Au moins depuis la moitié du XIX ${ }^{\text {ème }}$ siècle, on a posé des questions sur le thème de l'évaluation archivistique. Le point central des préoccupations était, alors, la question des espaces occupés par les expressives masses de documents gouvernementaux (et leurs conséquents coûts de maintien). Il était donc nécessaire de définir ce qu'on doit préserver et ce qu'on doit éliminer. Et, d'après un bilan effectué à ce propos par Carol Couture (1996-1997, p. 3-31), on peut affirmer que, si dans la première moitié du XXème siècle, l'évaluation, grosso modo, tantôt s'est penchée sur la logique de production des documents de l'organisme générateur, tantôt a mis en valeur les demandes des usagers extérieurs aux organismes. Cependant, dans la seconde moitié du XXème siècle, de nouveaux éléments ont été ajoutés au débat; la relation de l'évaluation avec la perception de l'histoire contemporaine et avec les perspectives de construction de la connaissance historique a eu un poids significatif dans cette discussion, particulièrement intense entre les archivistiques d'Amérique du Nord, avec ceux qui proposent la « stratégie de documentation » (EUA) ou la « macro-évaluation » (Canada). 
L'un des points les plus marquants et polémiques qui ressortit de ce débat archivistique est la considération que la documentation gouvernementale, quelque volumineuse et pertinente qu'elle soit, n'est pas suffisante dans le travail d'évaluation réalisé par l'institution archivistique publique. Autant dans la perspective de ce qu'on appelle la «stratégie de documentation », proposée par des archivistes nord-américains dans les années 1980, autant dans celle de la «macro-évaluation » canadienne, l'évaluation ne devrait pas se préoccuper d'identifier ce qui serait significatif dans la documentation d'une administration publique donnée, sinon de détecter ce qui serait important pour la société dans laquelle cette administration serait insérée. D’après cette conception de la «stratégie de documentation ", l'évaluation ne devrait pas partir des documents qui existent déjà dans l'institution, mais d'une problématisation autour de ce qui sera documenté (SAMUELS, 1986, p.114-121). L'archiviste canadien Terry Cook, en commentant l'expérience de la macro-évaluation, a souligné qu'elle ne partirait pas de l'analyse des documents, mais des fonctions ou compétences générales des institutions qui les ont produits. Telles fonctions institutionnelles seraient prises comme des espèces de «filtres de tendances sociales, activités, besoins et souhaits, de choses et concepts que “valorise” la société (COOK, 1999, p.8-9). Dans ce sens, l'évaluation des documents publics, en plus d'être faite selon des critères extérieurs à elle, devrait être complétée par l'évaluation et incorporation, aux fonds de l'institution archivistique responsable de la garde, de documents d'archives d'autres provenances, ainsi que de collections à plusieurs caractéristiques, à condition qu'ils observent les critères adoptés. ${ }^{9}$

Sans approfondir ici la présentation de ces propositions ou discuter plus profondément la question de l'évaluation archivistique, ce que j'ai essayé de faire dans un autre travail (GONÇALVES, 2005, esp. p. 84-92), je me limite à signaler que, si la « stratégie de documentation» et la «macro-évaluation» ont la qualité de mettre en cause la relation des archives avec la dimension de la mémoire sociale et de la construction de l'histoire, elles présentent l'inconvénient de rendre encore plus intriquées les attributions,

\footnotetext{
9 II convient de souligner que la discussion canadienne valorise un débat antérieur, qui avait été commencé par l'archiviste Hans Booms, en Allemagne Occidentale, pendant la décennie 1970. La traduction du texte original de Booms en anglais date de 1987 et a été divulguée par la revue Archivaria; la traduction en français a été publiée dans la revue Archives de 2001-2002 (BOOMS, 2001-2002, p. 7-44). La revue Archives a publié aussi un autre texte de Booms sur l'évaluation, en 2003-2004 (BOOMS, 2003-2004, p. 19-28).
} 
déjà très amples et complexes, comme celles des institutions archivistiques en face de l'évaluation de documents. Elles tendent aussi à rapprocher l'ensemble institutionnel de documents des centres de documentation (ou plutôt des « centres de mémoire »?). Elles s'éloignent, ainsi, du profil concret de l'ensemble archivistique de référence pour se mettre en quête de ce qui est «historiquement représentatif » sur et pour la société (quelque chose certainement très difficile à caractériser et sujet à une infinité de contestations, car basé sur une interprétation qui s'éloigne des spécificités de l'ensemble archivistique - voir (AMARGO, 2003, surtout p. 14-15). Elles s'ouvrent, encore, à l'«instrumentalisation politique » du travail archivistique, comme a montré Angelika Menne-Haritz (MENNE-HARITZ, 1994, p. 535). En plus, ces propositions tendent à laisser de côté la dimension de la société qui est présente à la documentation archivistique publique elle-même (dimension produite dans les interactions entre citoyens et gouvernements, comme nous avons discuté plus haut) et à valoriser peu l'articulation entre documents publics et le nécessaire compte rendu des gouvernements.

La demande pour transparence est croissante, en ce qui concerne les actions des gouvernements, ce qui est démontré par le recours toujours plus amplifié aux outils de gouvernement électronique, dans plusieurs pays, et de façon spectaculaire, en dehors des instances gouvernementales, par WikiLeaks. Dans le Gouvernement fédéral brésilien, la préoccupation avec la transparence est plus directement dirigée vers le contrôle de l'application de ressources publiques, comme on peut voir sur le site «Portal da Transparência do Governo Federal » - <http://www.portaltransparencia.gov.br>. Cette préoccupation se multiplie dans des gouvernements d'états de la fédération et de villes, avec la création de sites similaires, surtout après l'approbation de la loi fédérale $n$. 12.527/2011. Dans la société civile, il y a aussi des initiatives importantes, comme celle de l'association «Transparência Brasil », qui a pour objectif de combattre la corruption, en accompagnant principalement la réalisation d'appels d'offres, le financement de campagnes électorales et le travail de parlementaires et ministres du Supremo Tribunal Federal: http://www.transparencia.org.br. Mais il faut souligner que les documents d'archives gouvernementaux, générés en papier ou électroniquement, sont la base de cette transparence, comme l’a aussi démontré WikiLeaks. 


\section{Considérations finales}

À titre de considérations finales, il conviendrait encore de mettre en valeur deux questions : comment éviter que documents d'intérêt public soient déniés aux citoyens (ce qui est fait en dépit de la loi et du rôle des institutions archivistiques)? Les institutions archivistiques peuvent-elles ou doivent-elles éviter ce refus, en étant surtout plus agiles à rendre disponibles les documents sous sa garde?

La garantie d'accès aux documents publics est liée à des dispositifs légaux, mais aussi à des conditions d'organisation et conservation physique des documents. Quant à l'organisation et à la conservation physique, il appartient aux institutions, en vue des demandes sociales pour transparence, du droit à l'information et du droit à la mémoire, de disposer de ressources humaines et technologiques pour éviter que le traitement technique adéquat qui doit être donné aux documents empêche l'accès rapide et ample à la documentation. En ce qui concerne la législation portant sur l'accès, et notamment celle sur ce qui doit être confidentiel ou pas, et pour combien de temps, elle doit être accomplie par les institutions archivistiques. Si elle est jugée inadéquate, il convient de la contester et d'essayer de la modifier, ce qui doit certainement rassembler beaucoup d'efforts, non pas exclusifs des institutions archivistiques, et ni du domaine archivistique, car il s'agit d'une question qui concerne les intérêts de tous les citoyens. Cela peut être observé clairement dans le cas brésilien, compte tenu des progrès et des reculs dans cette question (WEICHERT, 2009, p.412-422), au moins depuis la loi fédérale n. 8.159/1991, et aussi la récente loi fédérale n. 12.527/2011, la « Loi d’accès aux informations ».

Il est impossible, cependant, de garantir convenablement l'accès à quelque chose qu'on ne contrôle pas, qu'on ne conserve pas, qu'on ne connaît pas, qu'on ne comprend pas : le traitement technique adéquat des ensembles de documents archivistiques est une condition fondamentale de leur accessibilité. II en résulte l'extrême importance de la gestion des documents, et aussi, particulièrement, l'importance des procédures de classement et évaluation des documents, tous supposant leur réalisation par un personnel préparé à des tâches si complexes (ce qui renvoie, inévitablement, à leur qualification et au problème de la formation des professionnels d'archives). Et, si l'accès 

accomplir leurs attributions. Garantir l'accès aux documents d'archives publics requiert, par conséquent, qu'on rende plus fortes les institutions archivistiques publiques : leur rôle social doit devenir plus clair et plus visible, en même temps qu'elles doivent être perçues socialement comme importantes et fiables (et, bien évidemment, être dignes de cette perception).

Dans ce sens, même si la législation empêche la consultation immédiate de documents classés comme confidentiels, il convient de poser la question : l'existence de ces documents doit être aussi confidentielle? Si la consultation à une documentation donnée est temporairement interdite, en raison de droits individuels à la protection de l'image, de la dignité et de l'honneur, sera-t-il convenable de se taire à propos d'elle? Sonia Combe (1994, p. 43-47), à propos des archives françaises, a démontré comment cette imposition de silence par rapport à ce qui est temporairement indisponible à consultation, résultant en des inventaires lacunaires, est nuisible aux chercheurs ou intéressés en général à la consultation à la documentation archivistique, faisant preuve d'un plus grand engagement des archivistes français avec la logique du secret d'Etat qu'avec celle du « compte rendu » à la société. Le plongement de Sonia Combe dans le monde des archives françaises a aussi fait qu'elle ait «la conviction que le mode de gestion de nos archives [en France] constitue un enclave de "totalitarisme" dans notre espace démocratique »(COMBE, 1994, p. 27). Si les institutions archivistiques sont décidemment engagées à la transparence, elles devraient présenter des informations générales sur tels documents encore indisponibles (soit parce qu'ils ne sont pas encore organisés, soit parce qu'ils ont besoin de soins de conservation, soit parce qu'ils sont classés comme confidentiels) : échéances, activités génératrices, données générales y contenues, démarches, documents qui les concernent, etc., quelque chose qui est nécessairement obtenu dans le processus de l'évaluation archivistique. Pourquoi, alors, ne pas essayer de garantir que le contrôle de la documentation produite, ses démarches, ses échéances de garde, sa destination, soient amplement divulgués ? 
Les technologies de l'information servent déjà à la gestion des documents, et il est important que les systèmes informatisés existants se préoccupent d'interagir avec les usagers de l'administration publique elle-même et avec les usagers-citoyens. II est fondamental que les «intéressés en général » soient envisagés non seulement par les archives publiques dans sa phase permanente, mais aussi dans toutes leurs phases. Comme l'épisode concernant WikiLeaks, ici relaté, fait voir, le réseau mondial d'ordinateurs est devenu un outil puissant de dissémination de l'information, et il appartiendrait aux institutions archivistiques publiques d'étendre et approfondir leur utilisation. Il est certain, cependant, que la grande plasticité des documents numériques et la facilité avec laquelle ils peuvent être modifiés, mutilés et tordus font objet de préoccupations additionnelles en ce qui concerne les soins qui doivent entourer leur divulgation dans ce gigantesque réseau électronique de communication et information. La nature des liens des institutions archivistiques publiques avec la documentation gouvernementale leur donne l'avantage de pouvoir sceller l'authenticité des informations fournies, car elles doivent connaître, en principe, la trajectoire de génération, démarches, évaluation, garde et la mise à disposition des propres documents.

Concilier gouvernance et transparence, ainsi que l'exercice de droits qui, dans une société démocratique, se heurtent assez souvent (comme, dans le cas des archives, le droit à l'information et le droit à l'inviolabilité de l'intimité, de la vie privée, de l'honneur et de l'image), c'est une tâche complexe qui met en relief la place des institutions archivistiques publiques et leur ensembles de documents. II appartient à ces institutions de chercher des réponses à la hauteur des défis, et aux citoyens de les exiger.

\section{Références}

ALBERCH FUGUERAS, R.; CRUZ MUNDET, J.R. ¡Archive-se! - los documentos del poder, el poder de los documentos. Madrid: Alianza Editorial, 1999.

ASSANGE, J. et al. Cypherpunks: liberdade e o futuro da internet. São Paulo: Boitempo, 2013. 
BLOCH, M. Apologia da história ou O ofício de historiador. Rio de Janeiro: Jorge Zahar, 2002.

BOOMS, H. La constitution du patrimoine archivistique: I'archivage comme activité sociale et politique. Archives, v. 35, n.1-2, 2003-2004, p.19-28. Disponível em: <http://www.archivistes.qc.ca/revuearchives/vol35_1_2/35-1-2-booms.pdf> Acesso em: 16 abr. 2013.

BOOMS, H. Ordre social et constitution du patrimoine archivistique: à propos de l'évaluation des sources d'archives. Archives, v. 33, n.3-4, 2001-2002, p.7-44. Disponível em: <http://www.archivistes.qc.ca/revuearchives/vol33_3-4/33-3-4-booms.pdf>. Acesso em: 16 abr. 2013.

CAIRE-JABINET, M.-P. Introdução à historiografia. Bauru (SP): EDUSC, 2003.

CAMARGO, A. M. de A. Sobre o valor histórico dos documentos. Arquivo Rio Claro. Rio Claro (SP): Arquivo do Município de Rio Claro, n.1, p.11-17, 2003.

CAMARGO, A. M. de A. Os arquivos e o acesso à verdade. In: SANTOS, C.M., TELES, E., TELES, J.de A. Desarquivando a ditadura: Memória e Justiça no Brasil. São Paulo: Hucitec, 2009. v.2, p.424-443.

CAMPANHOlE, A., CAMPANHOLE, H. L. (orgs.). Constituições do Brasil. 11 ed. São Paulo: Atlas, 1994.

CARBONELL, C.-O. La historiografia. México: Fondo de Cultura Económica, 1986.

COMBE, S. Archives interdites: les peurs françaises à l'Histoire contemporaine. Paris: Albin Michel, 1994.

COOK, T. Archival appraisal and collection: issues, challenges, new approaches [Special Lecture Series, University of Maryland and NARA Staff, 21-22 April 1999]. Disponível em: <http://www.mybestdocs.com/cookt-nara-990421-2.htm>. Acesso em: 12 abr.2013.

COOK, T. Arquivos pessoais e arquivos institucionais: para um entendimento arquivístico comum da formação da memória em um mundo pós-moderno. Estudos históricos, Rio de Janeiro: FGV, v.11, n.21, p.129-149, 1998.

COUTURE, C. L'évaluation des archives: état de la question. Archives, v. 28, n.1, 1996-1997, p.3-31. Disponível em: <http://www.archivistes.qc.ca/revuearchives/vol28_1/28-1couture.pdf $>$. Acesso em: 13 abr. 2013. 
DELMAS, B. La société sans mémoire: propos dissidents sur la politique des archives en France. Paris: Bourin, 2006.

DUCHEIN, M. Les obstacles à l'accés, à l'utilisation et au transfert de l'information contenue dans les archives: une étude RAMP. Paris: UNESCO, 1983.

FINDLAY, C. People, records and power: what archives can learn from WikiLeaks, Archives and Manuscripts. Sydney: Australian Society of Archivists, v.41, n.1, p.7-22, 2013. Disponível em: <http://dx.doi.org/10.1080/01576895.2013.779926 >. Acesso em 18 abr. 2013.

GINZBURG, C. Relações de força: história, retórica, prova. São Paulo: Companhia das Letras, 2002a.

GINZBURG, C. Sinais: raízes de um paradigma indiciário. In: _..Mitos, emblemas, sinais: morfologia e história. 2 ed. São Paulo: Companhia das Letras, 2002b.

GONÇALVES, J. Arquivos no presente: o lugar da História. Cadernos do CEOM, Chapecó: Centro de Memória do Oeste de Santa Catarina, n.22, p.75-96, dez.2005.

HARTOG, F. O tempo desorientado - tempo e história: "Como escrever a história da França?”. Anos 90. Porto Alegre: UFRGS, n.7, p.7-28, jul.1997.

HARTOG, F. Regimes de historicidade: presentismo e experiências do tempo. Belo Horizonte: Autêntica, 2013.

JARDIM, J. M. Transparência e opacidade do Estado no Brasil: usos e desusos da informação governamental. Niterói (RJ): Editora da UFF, 1999.

LE GOFF, J. História e memória. São Paulo: Ed. da Unicamp, 1996.

LAMBERT, P., HARRISON, R., JONES, Aled. Metodologia - história científica e o problema da objetividade. In: LAMBERT, P., SCHOFIELD, P. (orgs.). História - introdução ao ensino e à prática. Porto Alegre: Penso, 2011. p.43-55.

LODOLINI, E. Archivística: princípios y problemas. Madrid: ANABAD, 1993.

MENNE-HARITZ, A. Appraisal or documentation: can we appraise archives by selecting content? The American Archivist. Chicago: Society of American Archivists, v.57, p.528-542, 1994.

REIS, J. C. A história, entre a filosofia e a ciência. São Paulo: Ática, 1999. 
ROUSSO, H. O arquivo ou o indício de uma falta. Estudos históricos. Rio de Janeiro: FGV, v.9, n.17, 1996, p.85-91.

SAMUELS, H. W. Who controls the past? The American Archivist. Chicago: Society of American Archivists, v.49, n.2, p. 109-124, Spring 1986. Disponível em: <http://archivists.metapress.com/content/t76m2130txw40746/fulltext.pdf>. Acesso em: 18 abr. 2013.

SCHWARTZ, J. M.; COOK, T. Arquivos, documentos e poder: a construção da memória moderna. Registro. Indaiatuba (SP): Arquivo Municipal de Indaiatuba, n.3, p.18-33, jul.2004.

SILVA, A. M. da, et al. Arquivística - teoria e prática de uma ciência da informação. Porto: Afrontamento, 1999. v.1.

TESSITORE, V. Os Registros de Terras de São Paulo - inventário analítico. Revista Brasileira de História, São Paulo: ANPUH, v.6, n.12, p.187-196, mar./ago.1986.

VEYNE, P. Acreditaram os gregos nos seus mitos? [Lisboa]: Edições 70, 1987.

VIANA, N. O WikiLeaks e as batalhas digitais de Julian Assange. In: ASSANGE, J. et al. Cypherpunks: liberdade e o futuro da internet. São Paulo: Boitempo, 2013. p.9-18.

WEICHERT, M. A. Arquivos secretos e direito à verdade. In: SANTOS, C.M., TELES, E., TELES, J.de A. Desarquivando a ditadura: Memória e Justiça no Brasil. São Paulo: Hucitec, 2009. v.2, p.406-423. 